\title{
On the stability of laminar flow of a dusty gas
}

\author{
By P. G. SAFFMAN \\ Department of Mathematics, King's College, London
}

(Received 19 December 1961)

The equations describing the motion of a gas carrying small dust particles are given and the equations satisfied by small disturbances of a steady laminar flow are derived. The effect of the dust is described by two parameters; the concentration of dust and a relaxation time $\tau$ which measures the rate at which the velocity of a dust particle adjusts to changes in the gas velocity and depends upon the size of the individual particles. It is shown that if the dust is fine enough for $\tau$ to be small compared with a characteristic time scale associated with the flow, then the addition of dust destabilizes a gas flow; whereas if the dust is coarse so that $\tau$ is relatively large, then the dust has a stabilizing action.

For plane parallel flow, it is shown that the stability characteristics for a dusty gas are still determined by solutions of the Orr-Sommerfeld equation, but with the basic velocity profile replaced by a modified profile which is in general complex. A simple, although unrealistic, example is used to illustrate some features of the action of dust. It is intended to describe the solution of the modified Orr-Sommerfeld equation for plane Poiseuille flow in a later paper.

\section{Introduction}

The observation that adding dust to air flowing in turbulent motion through a pipe can appreciably reduce the resistance coefficient has been recently reported by Sproull (1961). A similar report that the aerodynamic resistance of a dusty gas flowing through a system of pipes is less than that of a clean gas has also been made by Kazakevich \& Krapivin (1958) (for a summary of this paper, see App. Mech. Rev. 1961, 14, §1117). These observations can be expressed as saying that the pressure difference required to maintain a given volume rate of flow is reduced by the addition of dust. It is to be noted that the increased density of a dusty gas should, all other things being equal, require a larger pressure difference to maintain a given volume flow rate. Sproull interprets the phenomenon as implying a substantial reduction in the value of the viscosity of a dusty gas, by as much as $40 \%$, compared with the clean gas. His explanation of this reduction is that the viscosity is proportional to the mean free path of the gas molecules and this is substantially reduced by the gas molecules colliding with the dust particles. However, his calculation of this effect is difficult to follow and, moreover, it contradicts the Einstein formula for the viscosity of a suspension (see, for example, Landau \& Lifshitz 1959, §22), according to which the viscosity of a dusty gas should be increased by a factor proportional to the concentration by volume of the dust particles. 
A more plausible explanation seems to be that the dust damps the turbulence. A dust particle in air, or any other gas, has a much larger inertia than the equivalent volume of air and will not therefore participate as readily in the turbulent fluctuations. The relative motion of the dust particles and the air will dissipate energy because of the drag between dust and air, and extract energy from the turbulent fluctuations. If, as certainly seems possible, the turbulent intensity is reduced then the Reynolds stresses will be decreased and the force required to maintain a given flow rate will likewise be reduced.

The problem of turbulence is related to the question of the stability of laminar flows and this suggested the present investigation of the effect of dust particles on the stability of a laminar flow of a gas, in order to see how dust may affect the critical Reynolds number for transition from laminar to turbulent flow. In the present paper, we shall be concerned with the equations of motion, some general results on the effects of fine and coarse dust, and the derivation of a modified Orr-Sommerfeld equation for the stability of plane parallel flow. A later paper, which is being written by Dr D. H. Michael, will present a detailed calculation of the stability characteristics of plane Poiseuille flow of a dusty gas.

\section{Equations of motion}

In order to formulate the problem in a reasonably simple manner and to bring out the essential features, we shall make simplifying assumptions about the motion of the dust particles. It will be supposed that the dust particles are uniform in size and shape, and that their velocity and number density can be described by fields $\mathbf{v}(\mathbf{x}, t)$ and $N(\mathbf{x}, t)$. We also assume that the bulk concentration (i.e. concentration by volume) of the dust is very small so that the net effect of the dust on the gas is equivalent to an extra force $K N(\mathbf{v}-\mathbf{u})$ per unit volume, where $\mathbf{u}(\mathbf{x}, t)$ is the velocity of the gas and $K$ is a constant, where it is also supposed that the Reynolds number of the relative motion of dust and gas is small compared with unity so that the force between dust and gas is proportional to the relative velocity. Then with small bulk concentration and the neglect of the compressibility of the gas, the equations of motion and continuity of the gas are

$$
\begin{gathered}
\rho\left(\frac{\partial \mathbf{u}}{\partial t}+(\mathbf{u} . \nabla) \mathbf{u}\right)=-\nabla p+\mu \nabla^{2} \mathbf{u}+K N(\mathbf{v}-\mathbf{u}), \\
\operatorname{div} \mathbf{u}=0
\end{gathered}
$$

where $\rho$ is the pressure less the hydrostatic pressure, and $\rho$ and $\mu$ are the density and viscosity of the clean gas. If the dust particles are spheres of radius $a$, $K=6 \pi a \mu$ by the Stokes drag formula.

As will be seen below, the effect of the dust is measured by the mass concentration, $f$ say. The bulk concentration is $f \rho / \rho_{1}$, where $\rho_{1}$ is the density of the material in the dust particles. For common materials, $\rho_{1} / \rho$ will be of the order of several thousand or more, so that the mass concentration may be a significant fraction of unity while the bulk concentration is small. It is to be noted that for suspensions in liquids, the bulk and mass concentrations will be roughly the same, so that qualitative differences in the motion of dusty gases and suspensions in liquids may be expected. For spherical particles, the Einstein increase in 
viscosity is $\frac{5}{2} \mu f \rho / \rho_{1}$, which is negligible for a dusty gas but may be significant for a liquid suspension.

The force exerted on the dust by the gas is equal and opposite to the force exerted on the gas by the dust, so that the equation of motion of the dust is

$$
m N\left(\frac{\partial \mathbf{v}}{\partial t}+(\mathbf{v} \cdot \nabla) \mathbf{v}\right)=m N \mathbf{g}+K N(\mathbf{u}-\mathbf{v})
$$

where $m N$ is the mass of dust per unit volume and $\mathbf{g}$ is the acceleration due to gravity. (The buoyancy force is neglected since $\rho / \rho_{1}$ is small.) The equation of continuity for the dust is

$$
\frac{\partial N}{\partial t}+\operatorname{div}(N \mathbf{v})=0 .
$$

Consider now a steady laminar flow in which the streamlines are straight and parallel. For sufficiently small particles, the velocity of sedimentation will be small compared with a characteristic velocity of the flow and can be neglected. Then in a steady state, the inertia terms in the equations of motion vanish identically and the dust particles move along the streamlines with the velocity of the gas, i.e. $\mathbf{v}=\mathbf{U}$, where $\mathbf{U}$ is the velocity of the gas. The number density $N$ is constant along the streamlines, and we shall suppose further that it has the constant value $N_{0}$ everywhere.

To investigate the stability of the basic flow to infinitesimal disturbances, we write $\mathbf{u}=\mathbf{U}+\mathbf{u}^{\prime}, \mathbf{v}=\mathbf{U}+\mathbf{v}^{\prime}, p=P+\rho p^{\prime}, N=N_{0}+N^{\prime}$, substitute into the equations, and linearize in the perturbations, to obtain

$$
\begin{gathered}
\frac{\partial \mathbf{u}^{\prime}}{\partial t}+\left(\mathbf{u}^{\prime} . \nabla\right) \mathbf{U}+(\mathbf{U} . \nabla) \mathbf{u}^{\prime}=-\nabla p^{\prime}+\nu \nabla^{2} \mathbf{u}^{\prime}+s\left(\mathbf{v}^{\prime}-\mathbf{u}^{\prime}\right) \\
\frac{\partial \mathbf{v}^{\prime}}{\partial t}+\left(\mathbf{v}^{\prime} . \nabla\right) \mathbf{U}+(\mathbf{U} . \nabla) \mathbf{v}^{\prime}=\left(\mathbf{u}^{\prime}-\mathbf{v}^{\prime}\right) / \tau \\
\operatorname{div} \mathbf{u}^{\prime}=0, \quad \frac{\partial N^{\prime}}{\partial t}+\operatorname{div}\left(N^{\prime} \mathbf{U}+N_{0} \mathbf{v}^{\prime}\right)=0
\end{gathered}
$$

Here, $\nu=\mu / \rho$ is the kinematio viscosity of the clean gas, $s=K N_{0} / \rho$ has the dimensions of frequency, and $\tau=m / K$ may be called the relaxation time of the dust particles. It is a measure of the time for the dust to adjust to changes in the gas velocity. For spherical particles of radius $a$,

$$
\tau=\frac{4}{3} \pi a^{3} \rho_{1} / 6 \pi a \mu=\frac{2}{9} \frac{a^{2}}{\nu} \frac{\rho_{1}}{\rho}
$$

Note that

$$
s \tau=m N_{0} / \rho=f .
$$

In the experiments reported by Sproull, the mass concentration was as large as $0 \cdot 2$.

\section{The cases of fine and coarse dust}

The effect of the dust enters through the two parameters $f$ and $\tau$. The former describes how much dust is present and the latter is determined by the size of the individual particles. Making the dust fine decreases $\tau$, and making it coarse 
increases $\tau$, in a manner proportional to the surface area of the particles. For the two extremes of $\tau$ relatively large or small, it is possible to simplify the equations, and we shall now examine these two limiting cases.

Let $L$ denote the length scale and $U^{*}$ the velocity scale of the basic flow. The known results of hydrodynamic stability theory lead to the belief that $L$ will also be, in general, the length scale of the most unstable disturbance. Consider now equation (6) for $\mathbf{v}^{\prime}$ in terms of $\mathbf{u}^{\prime}$. For a disturbance of length scale $L$, the lefthand side is of order $U^{*} \mathbf{v}^{\prime} / L$. The right-hand side is of order $\left(\mathbf{u}^{\prime}-\mathbf{v}^{\prime}\right) / \tau$.

Hence if $\tau \ll L / U^{*}$, which will be so if the dust is sufficiently fine, it follows that $\mathbf{v}^{\prime} \doteqdot \mathbf{u}^{\prime}$ for disturbances with length scale $L$ or larger. Then (6) gives

$$
\mathbf{u}^{\prime}-\mathbf{v}^{\prime} \doteqdot \tau\left(\frac{\partial \mathbf{u}^{\prime}}{\partial t}+\left(\mathbf{u}^{\prime} \cdot \nabla\right) \mathbf{U}+(\mathbf{U} . \nabla) \mathbf{u}^{\prime}\right)
$$

Substituting into equation (5) and using (9), we obtain

$$
\frac{\partial \mathbf{u}^{\prime}}{\partial t}+\left(\mathbf{u}^{\prime} . \nabla\right) \mathbf{U}+(\mathbf{U} . \nabla) \mathbf{u}^{\prime}=-\nabla \frac{p}{1+f}+\frac{\nu}{1+f} \nabla^{2} \mathbf{u}^{\prime}
$$

Thus the effect of fine dust for which $\tau \ll L / U^{*}$ is to make the flow analogous, as far as disturbances of length scale $L$ or larger are concerned, to that of a clean gas with the same basic velocity distribution but with a smaller kinematic viscosity. The physical reason for this is clear. For if $\tau$ is small compared with the time scale of the disturbance, it follows that the dust moves with the gas so that the effect of the dust is simply equivalent to increasing the density of the gas. Since the viscosity is sensibly unaffected by the dust, the effective kinematic viscosity is reduced by the factor by which the density is increased, namely $1+f$.

Suppose now that $\tau \gg L / U^{*}$, which will be so if the dust is sufficiently coarse or the flow sufficiently fast, then it follows that, for disturbances of time scale $L / U^{*}$ or smaller, the dust perturbation velocity $\mathbf{v}^{\prime}$ is negligibly small. Equation (5) then reduces to

$$
\frac{\partial \mathbf{u}^{\prime}}{\partial t}+\left(\mathbf{u}^{\prime} . \nabla\right) \mathbf{U}+(\mathbf{U} . \nabla) \mathbf{u}^{\prime}=-\nabla p^{\prime}+\nu \nabla^{2} \mathbf{u}^{\prime}-s \mathbf{u}^{\prime} .
$$

Thus the effect of the dust is now equivalent to an extra frictional force proportional to the velocity. If $\sigma$ and $\sigma^{*}$ are the amplification factors of a disturbance of given wavelength for the dusty and clean gas, respectively, it follows from (11) that $\sigma=\sigma^{*}-s$. The criterion for neutral stability of the disturbance is $\sigma=0$, i.e. $\sigma^{*}=s$. Thus coarse dust will increase the critical Reynolds number for the existence of a neutral disturbance of given wavelength. The physical explanation is that coarse dust does not move with the gas when the flow is perturbed but carries on with the velocity of the basic flow. The disturbance has therefore to flow around the particles, and energy is dissipated. This energy is extracted from the kinetic energy of the disturbance which is thereby stabilized.

There seems to be no way in which the presence of the dust can create extra modes of instability, as is the case for instance for flow past a flexible boundary (Brooke Benjamin 1960) where resonance between waves in the fluid and waves in the boundary produces extra modes of instability which are absent if the walls 
are rigid. As far as can be seen at present, the dust only modifies the waves which exist in a clean gas. We may therefore expect that the length scale of the neutral disturbance at the critical or minimum Reynolds number for instability is still of order $L$ for a dusty gas. It then follows that for fine dust with $\tau \ll L / U^{*}$, the critical Reynolds number (based on the dimensions of the velocity field and the kinematic viscosity of the clean gas) is reduced by the factor $1+f$. Thus fine dust destabilizes the flow.

On the other hand, coarse dust increases the critical Reynolds number and stabilizes the flow. The stabilizing effect can apparently be quite substantial for quite small values of $f$. Consider, for example, plane Poiseuille flow. Values of $\sigma^{*}=\alpha c_{i}$ have been calculated for various Reynolds numbers $R=U^{*} L / \nu$ (see Lin 1955, figure 3.1). Remembering that our $\alpha c_{i}$ is dimensional, we can express the results in the form $\alpha c_{i}=U^{*} g(R) / L$, where $g(R)$ is dimensionless and is partially given in Lin's book. The critical Reynolds number is then given by the least value of $R$ which satisfies $U^{*} g(R) / L=s=f / \tau$. With a value of $\tau U^{*} / L=5$, a value $f=3 \times 10^{-2}$ increases the critical Reynolds number from 5780 to about 16,000 . If, as may be possible, $g(R)$ has a maximum value, the addition of a sufficient amount of coarse dust may stabilize the flow completely towards infinitesimal disturbances.

It should be noted that the stabilizing action of coarse dust with $\tau \gg L / U^{*}$ depends only upon the parameter $s=f / \tau$. Thus if $f$ is kept constant, increasing the size of coarse dust particles decreases $s$ and reduces the stabilizing effect. The implication is that, for given $f$, there is a value of $\tau$ for which the stabilizing effect is maximized; and for values of $\tau$ greater than this particular value it is not correct to say that the coarser the dust, the more stable the flow. This is not paradoxical, because if $\tau$ becomes large for fixed $f$, it means that the number of particles is decreased; and the implication is that the decrease in the number of particles more than outweighs the increase in the size of the individual particles when the size is relatively large.

To obtain an idea of the numerical values of $\tau$, consider air with $\nu=0 \cdot 15$ $\mathrm{cm}^{2} / \mathrm{sec}$ and take $\rho_{1} / \rho=4 \times 10^{3}$. Then for spherical particles of radius $a$, $\tau \doteqdot 6 \times 10^{3} a^{2} \mathrm{sec}$ with $a$ in $\mathrm{cm}$. With $a=10^{-3} \mathrm{~cm}, \tau \doteqdot 6 \times 10^{-3} \mathrm{sec}$; with $a=5 \times 10^{-3} \mathrm{~cm}, \tau \doteqdot 0 \cdot 15 \mathrm{sec}$.

The present analysis does not hold if the flow is not unidirectional, e.g. Couette flow between rotating cylinders, or if the velocity of sedimentation is not negligible, for then it is not true that $\mathbf{v}=\mathbf{U}$ in the basic flow and also the number density will not be constant. Nevertheless, the physical arguments that fine dust destabilizes and coarse dust stabilizes should still be valid. The detailed analysis of non-parallel flows is much more difficult than that for parallel flows.

It is appropriate to mention here that although in practice the dust particles may be of a range of sizes, this does not present serious difficulties. Provided the interaction between dust particles of different sizes is negligible, which will be so if the bulk concentration is very small, an equation of type (6) will hold for each species of particle and the last term on the right-hand side of (5) will be replaced by a sum of similar terms, one for each species. For fine particles, $\mathbf{v}^{\prime} \doteqdot \mathbf{u}^{\prime}$ and their effect is to increase the density; whereas coarse particles will 
have $\mathbf{v}^{\prime} \doteqdot 0$ and will produce a frictional resistance. In the general case of $\tau$ neither large nor small compared with $L / U^{*},(6)$ can be solved to give $\mathbf{v}^{\prime}$ in terms of $\mathbf{u}^{\prime}$, as described in the next section, for each species which can then be substituted into the appropriate term of (5).

\section{The Orr-Sommerfeld equation for plane parallel flow}

Consider plane parallel flow of a dusty gas where the streamlines are perpendicular to the $y$-axis, say, and the velocity is a function only of $y$. We still suppose that any velocity of sedimentation is negligible and that the concentration of the dust is uniform. The equations satisfied by small disturbances to the flow are linear in the perturbation, and the general disturbance can be regarded as a sum of Fourier components propagating in various directions in the $(x, z)$-plane. Squire (1933) pointed out that the neutral disturbance at the critical Reynolds number is two-dimensional in form, i.e. it is a Fourier component propagating in the direction of the basic flow. This result is for incompressible fluids, and it is known (see, for example, Lin 1955) that it is not true for compressible gases. We now investigate whether it is true for dusty (but incompressible) gases.

Consider a Fourier component of wave-number $\alpha$ which propagates with (complex) velocity $c$ and take the $x$-axis in the direction of propagation. Then in this coordinate system, the basic flow has components $(U(y), 0, W(y))$, and the small disturbance terms are independent of $z$ and vary with $x$ and $t$ like $e^{i \alpha(x-c t)}$. The components of equation (6) now become (for brevity, the accents are dropped henceforth)

$$
\begin{aligned}
i \alpha(U-c) v_{x}+v_{y} D U & =\left(u_{x}-v_{x}\right) / \tau, \\
i \alpha(U-c) v_{y} & =\left(u_{y}-v_{y}\right) / \tau, \\
i \alpha(U-c) v_{z}+v_{y} D W & =\left(u_{z}-v_{z}\right) / \tau,
\end{aligned}
$$

where $D \equiv d / d y$. These equations can be solved for the three components of $\mathbf{v}$ to give

$$
\left.\begin{array}{c}
v_{x}=\frac{u_{x}}{1+i \alpha \tau(U-c)}-\frac{\tau u_{y} D U}{\{1+i \alpha \tau(U-c)\}^{2}}, \quad v_{y}=\frac{u_{y}}{1+i \alpha \tau(U-c)}, \\
v_{z}=\frac{u_{z}}{1+i \alpha \tau(U \overline{-c})}-\frac{\tau u_{y} D W}{\{1+i \alpha \tau(U-c)\}^{2}} .
\end{array}\right\}
$$

We now substitute these expressions into the components of equation (5) and obtain after a little reduction

where

$$
\begin{aligned}
i \alpha(\bar{u}-c) u_{x}+u_{y} D \bar{u} & =-i \alpha p+\nu\left(D^{2}-\alpha^{2}\right) u_{x}, \\
i \alpha(\bar{u}-c) u_{y} & =-D p+\nu\left(D^{2}-\alpha^{2}\right) u_{y}, \\
i \alpha(\bar{u}-c) u_{z}+u_{y} D \bar{w} & =\nu\left(D^{2}-\alpha^{2}\right) u_{z},
\end{aligned}
$$

$$
\bar{u}(y)=U(y)+\frac{f\{U(y)-c\}}{1+i \alpha \tau\{U(y)-c\}}=U+\frac{f(U-c)}{1+\alpha^{2} \tau^{2}(U-c)^{2}}-\frac{i f \alpha \tau(U-c)^{2}}{1+\alpha^{2} \tau^{2}(U-c)^{2}},
$$

the second form being appropriate for the case of neutral stability when $c$ is real, and

$$
\bar{w}(y)=W(y)+f \int \frac{d W}{\{1+i \alpha \tau(\tilde{U}-c)\}^{2}} .
$$


The equation of continuity for the gas becomes

$$
i \alpha u_{x}+D u_{y}=0 .
$$

Equations (16), (17) and (21) are not coupled to (18) so that these three equations with appropriate boundary conditions for $u_{x}$ and $u_{y}$ determine the characteristic value problem for the unknown wave velocity $c$. Equation (18) is then an inhomogeneous equation for $u_{z}$, and when this is solved the velocity of the dust particles follows from (15). The remaining equation of continuity for the dust particles then determines the fluctuations of the dust concentration which does not enter into the above set of equations.

The component $W$ of the basic velocity does not enter into the three equations (16), (17) and (21) which determine $c$, and we therefore conclude that the behaviour of the three-dimensional disturbance is the same as that of a twodimensional disturbance (with $W=0$ and $u_{z}=0$ ) in a flow with a smaller basic velocity, since $U$ is the component, in the direction of wave propagation, of the basic velocity and is therefore less than the total basic velocity. It follows that for given $f, \tau$ and basic velocity profile, the neutral disturbance for the critical or smallest possible mean velocity is two-dimensional.

Three dimensionless parameters can be constructed out of the quantities defining the flow and the dust: the Reynolds number $R$, the mass concentration of dust $f$, and another one involving the relaxation time $\tau$ which it is convenient to take as $S=v \tau / L^{2}$. From the definition of $\tau$, it follows that $S$ is independent of the viscosity and it depends upon the relative size of the particles and their density relative to the gas; for spherical particles $S=\frac{2}{9}(\alpha / L)^{2}\left(\rho_{1} / \rho\right)$. Then for any three-dimensional neutral disturbance with given $f$ and $S$ there corresponds a two-dimensional neutral disturbance with smaller $R$, since $f$ and $S$ are independent of $U^{*}$ and the result of the previous paragraph applies. We therefore restrict attention to two-dimensional disturbances and take the $x$-axis in the direction of the basic flow.

We now introduce a stream function $\phi(y) e^{i \alpha(x-c t)}$ for $u_{x}$ and $u_{y}$ and eliminate the pressure between (16) and (17), to obtain in the usual manner the equation

$$
\left(D^{2}-\alpha^{2}\right)^{2} \phi=i \alpha R\left\{(\bar{u}-c)\left(D^{2}-\alpha^{2}\right) \phi-\left(D^{2} \bar{u}\right) \phi\right\},
$$

where we have here made all velocities and lengths dimensionless in terms of $U^{*}$ and $L$, and used the same symbol to denote corresponding dimensional and dimensionless quantities. The dimensionless form of (19) is

$$
\bar{u}(y)=U+\frac{f(U-c)}{1+i \alpha(U-c) S R}=U+\frac{f(U-c)}{1+\alpha^{2}(U-c)^{2} S^{2} R^{2}}-\frac{i f \alpha S R(U-c)^{2}}{1+\alpha^{2}(U-c)^{2} S^{2} R^{2}} .
$$

The boundary conditions on $\phi$ are $\phi=D \phi=0$ on rigid boundaries, and $\phi \rightarrow 0$ as $y \rightarrow \infty$ if the flow is unbounded in the $y$-direction. It is clear that (22) is the Orr-Sommerfeld equation with a new complex velocity $\bar{u}(y)$ replacing the ordinary fluid velocity $U(y)$. In other words, the effect of the dust is equivalent to a change in the velocity profile, with the added complication that the new velocity is complex. 
The results of the previous section about the effects of fine and coarse dust now follow from the limiting forms of (23) for the two cases of $S R=U^{*} \tau / L$ small or large compared with unity.

It may be mentioned here that the equivalence as regards stability of a dusty gas flow and a clean gas flow with a modified velocity profile holds generally for unidirectional flows and is not restricted to plane parallel flows. For a general unidirectional flow parallel to the $x$-axis, with the basic velocity now a function of $y$ and $z$ (for example, flow through a straight pipe), it is almost immediate that the equations for small disturbances of the velocity field which depend on $x$ and $t$ like $e^{i \alpha(x-c t)}$ are identical with those for a clean gas in which the basic velocity $\bar{u}(y, z)$ is given by (19) (or in dimensionless form by (23)) with $U=U(y, z)$.

Now the Orr-Sommerfeld equation is not easy to solve and there does not seem to be any simple but realistic flow situation which can be utilized without heavy computation to illustrate the effect of dust. It is intended to present results for plane Poiseuille flow, with $U(y)=1-y^{2}$ and rigid boundaries at $y= \pm 1$, in a later paper. However, it seems worth while to examine first a simple, although unrealistic, situation which has the virtue of bringing out clearly some of the main features of the effect of dust.

Let us consider for the sake of example the decay of disturbances in a semiinfinite volume of dusty gas bounded by a plane stress-free boundary $y=0$, say. We take the basic state as one in which the velocity and number density are uniform, so that with an appropriate choice of origin we have $U=0$. There is no basic length or velocity scale in the problem (which is why it can be solved without difficulty) so that it is more convenient to use dimensional quantities. The dimensional Orr-Sommerfeld equation for the disturbance of (dimensional) wave-number $\alpha$ is (22) with $R$ replaced by $1 / \nu$ and

$$
\bar{u}-c=-c\left(1+\frac{f}{1-i \alpha \tau c}\right)=-\bar{c}, \text { say. }
$$

The boundary conditions which express the vanishing of stress at the boundary are $D u_{x}=0$ and $-p+2 \nu D u_{y}=0$.

The form of $\phi(y)$ is $A e^{\alpha y}+B e^{\beta y}$, where $\beta=\left(\alpha^{2}-i \alpha \bar{c} / \nu\right)^{\frac{1}{2}}, \mathscr{R} \beta>0$, and the gas is supposed to be in the region $y<0$. The pressure follows from (16), and a straightforward application of the boundary conditions leads to $\bar{c}=\frac{1}{2} \nu \alpha( \pm \sqrt{ } 7-5 i)$. For small values of the mass concentration $f$, we can solve (24) by iteration and obtain, to the first order in $f$,

$$
c_{r}= \pm \frac{1}{2} \sqrt{ } 7 \nu \alpha\left(1-\frac{f}{1+8 \alpha^{4} \nu^{2} \tau^{2}}\right), \quad c_{i}=-\frac{5}{2} \nu \alpha+\frac{5}{2} \nu \alpha f \frac{1-\frac{16}{5} \alpha^{2} \tau \nu}{1-8 \alpha^{4} \nu^{2} \tau^{2}},
$$

where $c=c_{r}+i c_{i}$.

The value of $-\alpha c_{i}$ measures the rate of decay of the disturbance. Thus for small $\alpha^{2} \tau \nu$, the rate of decay of the disturbance is decreased by the factor $1-f \doteqdot(1+f)^{-1}$ in accordance with the result that fine dust destabilizes. On the other hand, when $\alpha^{2} \tau \nu$ is large, the rate of decay is increased by amount $f / \tau=s$, so that coarse dust stabilizes. The destabilizing effect of the increase in density balances the stabilizing effect of the extra dissipation when $\alpha^{2} \tau v=\frac{5}{16}$. The wave 
propagation velocity of the disturbance is decreased by the dust. For fixed $f$, the dust has its maximum stabilizing effect when $\alpha^{2} \tau \nu=\frac{5}{2}\left(1+\sqrt{\frac{13}{5}}\right)$. For fixed $f$ and $\tau$, the dust tends to destabilize the larger wavelengths with the larger time scales and to stabilize the smaller wavelengths for which the time scales are smaller.

I wish to thank Dr J. T. Stuart for the benefit of several most helpful discussions and his wide experience of hydrodynamic stability.

\section{REFERENCES}

Brooke Benjamin, T. 1960 J. Fluid Mech. 9, 513.

KaZAKkvioh, F. P. \& Krapivin, A. M. 1958 Izv. Vyssh. Uchebn. Zavedenïi. Energetica, no. $1,101$.

Landau, L. D. \& Limshitz, E. M. 1959 Fluid Mechanics. London: Pergamon.

LIN, C. C. 1955 The Theory of Hydrodynamic Stability. Cambridge University Press.

Sprould, W. T. 1961 Nature, 190, 976.

Squire, H. B. 1933 Proc. Roy. Soc. A, 142, 621. 